\title{
HTS Current Leads for the LHC
}

\author{
T. M. Taylor \\ CERN, Geneva, Switzerland
}

\begin{abstract}
Leads for transferring heavy current from power converters, working at room temperature, into the liquid helium environment required to operate superconducting magnets, have been long recognized as being an immediate application of the emerging technology of high temperature superconductivity. By correct dimensioning of such leads it should be possible to reduce significantly the cryogenic load which these leads represent. The Large Hadron Collider (LHC) currently under. construction at CERN is by far the largest user of superconducting magnets requiring the transfer of over 3 million ampères of current, and therefore has much to gain from the use of this technology. After a brief reminder of the motives and the outline of the project, a review will be given of the program to provide helium gas cooled current leads incorporating HTS sections.
\end{abstract}

\section{INTRODUCTION}

Particle physics beyond the domain explored with the Tevatron at Fermilab and the electron positron collider (LEP) at CERN calls for an energy range of $1 \mathrm{TeV}$ or more for the elementary constituents of matter, i.e. leptons or quarks. This cannot be achieved by upgrading LEP or by building larger circular $\mathrm{e}^{+}-\mathrm{e}^{-}$colliders, as the energy loss due to synchrotron radiation varies with the fourth power of the particle energy and becomes prohibitive for these light particles. Linear $\mathrm{e}^{+}-\mathrm{e}^{-}$ colliders are not limited by synchrotron radiation but are technically difficult, and at this moment appear to be prohibitively expensive to build.

At the present time the goal is best achieved by accelerating and colliding protons. Though still emitted, the synchrotron radiation is much less, because of the 2000 times larger proton mass, and acceleration is only limited by the maximum magnetic guiding field and the proton trajectory radius. With superconducting magnets cooled using superfluid helium and producing some $8.3 \mathrm{~T}$, it will be possible to accelerate and collide $7 \mathrm{TeV}$ proton beams in the existing LEP tunnel. This is the main motivation for the Large Hadron Collider (LHC) Project [1], which was approved by the CERN Council in December 1994. The LHC has now entered into the construction phase, and commissioning is foreseen for 2005 .

Manuscript received September 13, 1998
In order to excite the guiding and focusing magnets for this accelerator a total electrical current of about 3.5 MA must pass from the power converters, at room temperature, through the insulating vacuum and into the liquid helium environment. With classical vapour cooled leads a power of $1.1 \mathrm{~W} / \mathrm{kA}$ is dissipated in the liquid. By using high temperature superconductor (HTS) in the lower part of the lead a gain of a factor of ten is possible, giving a substantial saving in the cryogenic load.

\section{OUTLINE OF THE LHC}

After removal of the LEP machine, the LHC will be installed in the existing tunnel of $27 \mathrm{~km}$ in circumference. Its configuration is shown in Fig. 1. Eight identical $2.9 \mathrm{~km}$ long circular arcs house the superconducting magnets. The arcs are separated by long straight sections at the centre of four of which the two counter-rotating beams cross. The beams are focused and brought into collision at the interaction points (IPs) using special "insertion" magnets, where the collisions will be studied by means of large and sophisticated experiments installed at the IPs. Other long straight sections are devoted to machine utilities.

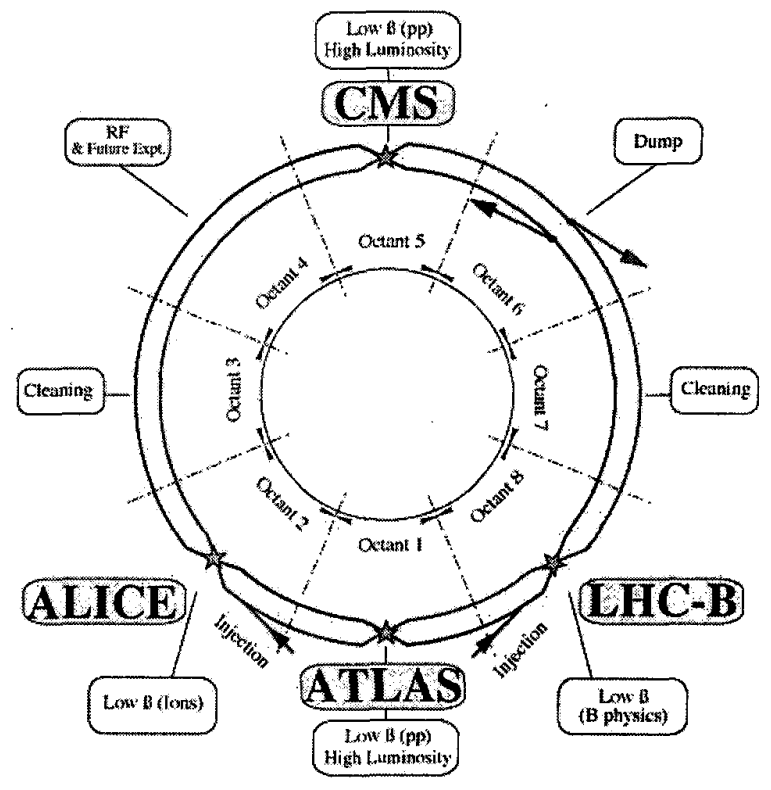

Fig. 1. Overall layout of the LHC machine 


\section{A. Magnets}

Hadron colliders require that beams travel into two separate channels and be guided by magnetic fields of equal strength but opposite in direction. To adapt to the available space in the existing tunnel (and minimise the cost), the two beam channels and their associated sets of coils are inserted in a common magnetic yoke and share the same mechanical structure and cryostat in a two-in-one configuration. The magnets in the LHC arcs consist of a periodic arrangement of three dipoles for guiding the beams followed by a short straight section which house a quadrupole for beam focusing, corrector magnets, and cryogenic equipment. Other dipoles and quadrupoles link the arcs to the long straight sections.

The 1232 dipoles, each of a magnetic length of $14.3 \mathrm{~m}$, and weighing about 32 tonnes are connected in series, octant by octant, each circuit having a stored energy of $1.25 \mathrm{GJ}$ at the maximum (ultimate) field level of $9 \mathrm{~T}$ at which the magnets are designed to operate. This magnet operates at up to $13 \mathrm{kA}$.

Focusing of the beams in the arcs is obtained by means of 396 twin aperture quadrupole magnets, also connected in series, but in two circuits per octant, one for each type ( $F$ or D). The magnets produce a field gradient of up to $240 \mathrm{~T} / \mathrm{m}$. These magnets also operate at up to $13 \mathrm{kA}$

Focusing in the dispersion suppressors and in the matching sections, which provides the small transverse beam dimensions required locally at the interaction point, is obtained by means of special quadrupoles excited individually or in small families. These magnets operate at up to $6 \mathrm{kA}$.

Hadron colliders are very sensitive to the quality of the magnetic field in the main magnets, and numerous correction magnets and families of magnets are provided for the control of beam orbits and other specific beam parameters such as chromaticity. These magnets are much smaller and operate at standardized currents of up to 60,120 or $600 \mathrm{~A}$.

A summary of the present tally of the circuits to be considered is given in Table I. The discharge time of the various circuits is also included in the table. In order to discharge the main dipole and quadrupole circuits in the times shown, and to extract safely the stored energy, an external resistance must be switched into the centre of the circuit. For this reason each dipole circuit has 4 leads. Moreover, most two $6 \mathrm{kA}$ circuits are configured to share 3 leads. Circuits will be tested at the withstand voltage to ensure that the insulation is such that arcing will not occur during quenching.

TABLE I

PRINCIPAL MAGNET CIRCUITS

\begin{tabular}{cccc}
\hline $\begin{array}{c}\text { Current } \\
(\mathrm{kA})\end{array}$ & Number & Discharge Time (s) & $\begin{array}{c}\text { Withstand Voltage } \\
(\mathrm{kV})\end{array}$ \\
\hline 13 & 8 & 105 & 3.5 \\
13 & 16 & 50 & 3.5 \\
13 & 4 & $<10$ & 2.5 \\
8 & 4 & $<10$ & 2.5 \\
6 & 212 & $<10$ & 2.5 \\
0.6 & 544 & $<10$ & 2.5 \\
\hline
\end{tabular}

\section{B. Cryogenics}

More than 40,000 tons of materials all around the LHC ring have to be cooled down to $1.9 \mathrm{~K}$. This temperature level, rather than the more conventional $4.5 \mathrm{~K}$, has been chosen as it allows the maximum attainable dipole field to be increased by some $2.6 \mathrm{~T}$, In addition, superfluid helium has other advantages such as the absence of viscosity, very large specific heat and heat conductivity, which taken together largely balance the technical problems associated with its use.

Eight large cryoplants will provide an equivalent refrigeration capacity of some $18 \mathrm{~kW}$ at $4.5 \mathrm{~K}$ each. After expansion in order to obtain the superfluid at $1.9 \mathrm{~K}$, the helium gas is brought up to atmospheric pressure at cryogenic temperatures using cold compressor boxes located in underground caverns. Interconnecting boxes will allow two cryoplants to be coupled, providing redundancy and speeding the cooling process if necessary.

A compound cryogenic distribution line will connect each cryoplant to the cryomagnets in the arc and supply them with helium at the required temperatures and pressures. As shown in Fig. 2, this runs alongside the cryomagnets. Within the cryoline, of outer diameter of $600 \mathrm{~mm}$, the large $20 \mathrm{~K}$ gas recovery line serves as a helium buffer in case of a quench of several magnets. Other lines serve to supply helium at $4.5 \mathrm{~K}, 3.6 \mathrm{bar}$, for filling the magnets, and gaseous helium at $50 \mathrm{~K}$ for the thermal screen. The cryoline is connected to the magnets of the arc at $106 \mathrm{~m}$ intervals, through a U-shaped composite line, which feeds the technical service module next to every other short straight section. Once the magnets are filled with liquid helium at $4.5 \mathrm{~K}$ and 1.1 bar, superfluid helium at $1.9 \mathrm{~K}$ is created in the service module by connecting it to the pumping line, and then sent in a linear heat exchanger running inside the magnets. This allows them to be operated in a static bath of superfluid helium at 1.1 bar. The distribution feed boxes (DFBs) through which the current is introduced at the ends of the arcs, are also connected to this cryoline.

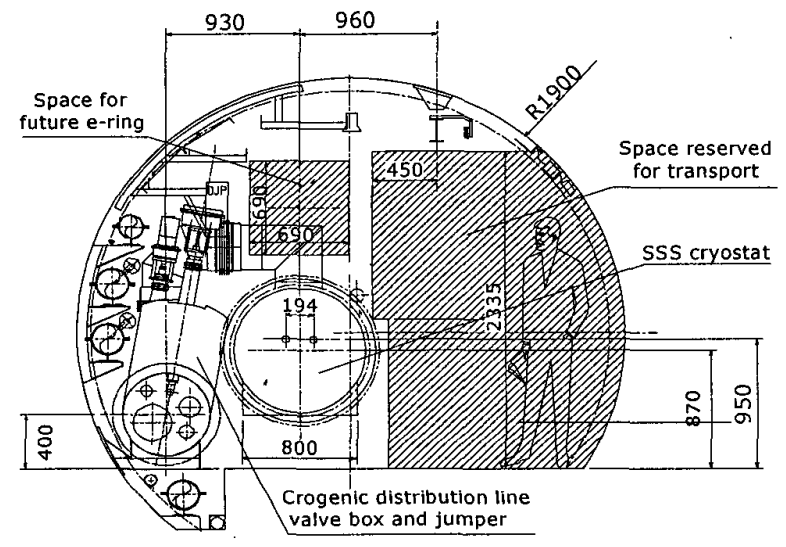

Fig. 2. Standard cross section of the LHC tunnel 


\section{Vacuum}

The LHC includes two vacuum systems: the insulation vacuum and the ultra high vacuum for the beam channels. The space between the magnet cold masses and the cryostat outer tanks is put under moderate vacuum in order to avoid heating the cryomagnet by conduction and by convection.

The required luminosity lifetime implies that the beams vacuum stays in the range of $10^{-9}-10^{-10}$ Torr. This should normally not be a problem, as the beams circulate in the cold bores which are at $1.9 \mathrm{~K}$ and are then very effective cryopumps. The circulating beams radiate quite some power, however, the total synchrotron radiation emitted from the two proton beams at $7 \mathrm{TeV}$ amounting to $0.4 \mathrm{~W} / \mathrm{m}$. In addition, beam image currents circulating in the vacuum chamber wall add resistive losses. These two effects would be an excessive load to the cryogenic system and a beam screen, cooled via a separate helium circuit, will be inserted in the cold bore to intercept the beam emitted power at $4.5-20 \mathrm{~K}$. This provides an abundant source of helium gas at $20 \mathrm{~K}$.

\section{Powering and Controls}

In operation all the dipole magnets of an arc are powered in series to a current of up to $13 \mathrm{kA}$, but the required voltage is low and determined essentially by the desired rate of rise of current. Power converters will be installed close to the magnets, in underground caverns and service tunnels at the even points, to minimise the voltage drop along warm bus work. Other magnets such as the lattice quadrupoles and harmonic correctors will also be powered in series, with superconducting busbars running through the magnet cold masses. The connection of all magnet circuits to their associated power converter requires a large number of current leads, which, with the exception of the low current (60 and 120 A) orbit correction dipole magnets, will be grouped at the ends of the arcs in distribution feed boxes (DFBs). Because of the very large energy stored in the magnets (7 MJ in each dipole), an elaborate magnet protection system must be provided, to avoid damage in case of a sudden resistive transition (quench); this will be based on cold diodes installed at each magnet.

In all transverse directions, both proton beams are surrounded by more than $300 \mathrm{~mm}$ of steel, which provide a relatively effective shielding against radiation. It is therefore estimated that at floor level we can expect a dose lower than 2$3 \mathrm{~Gy}$ per year of LHC running at full luminosity, and it will be possible to install in the tunnel most of the control electronics and low current power converters. This applies in particular to the quench protection detection and control, vacuum control, beam monitoring front end electronics, closed orbit correction, etc. All this equipment will be housed in small crates located under the central dipole and linked with the central control system through optical fibres. The protection diodes and the current leads, being nearer the beam axis, may receive a dose of the order of $100 \mathrm{~Gy}$ per year.
In the vicinity of the interaction points the final focus (inner quadrupole triplet) system will be subjected to the radiation emanating from the colliding particles, which is intense, and associated electronics will have to be installed in local shielded caverns. Moreover, the equipment to be installed in the tunnel, besides being sufficiently radiation hard, must be designed with the effect of induced radiation in mind.

\section{THE CURRENT LEADS}

\section{A. Choice of Cooling Method}

The current leads are designed to take full advantage of the cryogens available. The superconducting bus from the magnet traverses a lambda plate to enter the $4.5 \mathrm{~K}$ liquid helium bath in the DFB. At its cold end the lead dips into the $4.5 \mathrm{~K}$ helium. The lower section of the lead incorporates HTS material, the warm end of which is maintained at less than $50 \mathrm{~K}$ using helium gas from the $20 \mathrm{~K}, 1.3$ bar line. This gas is used to cool the classical upper, heat exchanger, section of the lead, and is recovered at room temperature in the warm gas line.

Given the quantities of gas available at the different temperatures, this cooling regime fits well into the cryogenic system of the LHC; it also provides very favourable conditions for operating HTS materials such as $\mathrm{BSCCO}$ and YBCO. A typical lead with its cooling is shown schematically in Fig. 3, and the basic functional requirements for the cooling of the current leads are presented in Table II.

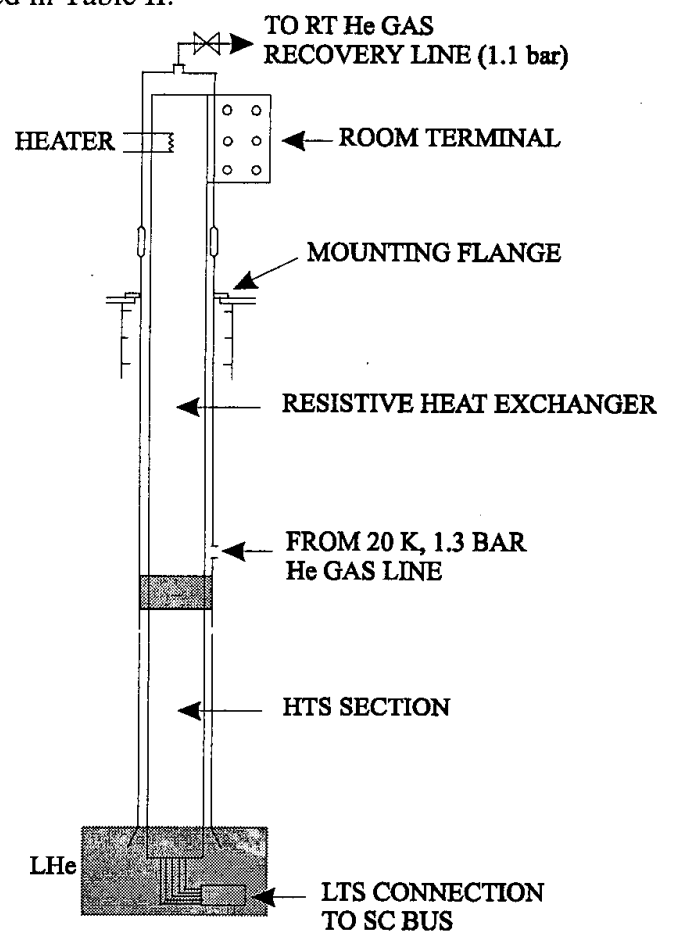

Fig. 3. A lead with its cooling scheme 
TABLE II

TARGET SPECIFICATIONS FOR THE COOLING OF THE LEADS

\begin{tabular}{lccc}
\hline $\begin{array}{l}\text { Cooling requirements } \\
\text { (maximum valves) }\end{array}$ & \multicolumn{3}{c}{ Lead Type } \\
\hline & $13 \mathrm{kA}$ & $6 \mathrm{kA}$ & $0.6 \mathrm{kA}$ \\
At zero current (standby) & & & \\
$20 \mathrm{~K} \mathrm{He}$ gas flow (g/s) & 0.5 & 0.25 & 0.03 \\
heat load into LHe (W) & 1 & 0.4 & 0.03 \\
At nominal current & & & \\
20 K He gas flow (g/s) & 1 & 0.5 & 0.06 \\
heat load into LHe (W) & 1.5 & 0.6 & 0.06 \\
\hline
\end{tabular}

Calculations, supplemented by tests, have shown [2] that it should be feasible to get a reduction of a factor of 10 in the dissipation of heat in the liquid helium, as compared to the classical case of cooling the lead with vapour boil off [3].

\section{B. Design Challenges}

While current leads are an obvious field of application for the new HTS materials, leads carrying current of the level required in the $\mathrm{LHC}$, other than a few research devices, have yet to be made. The design challenges include the development of rugged material, the making of sufficiently low resistance joints at the top and especially at the bottom of the HTS part, and the provision of sufficient insulation between lead and ground to allow the voltage testing of installed magnets. In addition, the integration of the HTS section into a full lead, including an optimised upper section, which can be handled safely by equipment riggers in the confined environment of the tunnel calls for serious engineering design work. Finally there is the question of stability in time. These materials have only recently become available; the early specimens were prone to degradation, and while it is claimed that this problem has been overcome, it remains a cause for concern, as we require leads which will perform satisfactorily for 10 to 15 years. Accelerated life tests are required to verify long term stability.

\section{Strategy for Obtaining Leads}

Although CERN does not have the resources available to make its own leads, it does have considerable expertise in the design and qualification of such equipment [2]. We have also gained experience over the last five years in the testing of HTS materials in various configurations. There is however great interest among prospective vendors, and it has therefore been decided to encourage interested companies by issuing specifications, based on our own calculations, for the supply of prototype leads which will be tested and characterized in a dedicated test rig at CERN. As the development of HTS materials is in constant progress, we have not insisted on any particular technology at this stage. The first specification to be issued was for the most difficult lead, for the main $13 \mathrm{kA}$ dipole circuits. There has been a good response, and one pair of leads has already been received and tested, with very encouraging results. After acceptance testing, life testing will be done on the most promising leads. It should be said that the upper "classical" heat exchanger section of the lead is also quite challenging, and the prototypes being supplied incorporate widely differing solutions. As the heat exchanger can be an expensive component, there is hope that creative thought in this direction will offset the added cost of the HTS section.

On the basis of results of the prototype testing, a precise specification will be issued for the supply of the series units. Thanks to encouragement through the prototyping exercise, we expect to interest a number of vendors, and that the prices will be competitive. The present layout calls for about $8013 \mathrm{kA}, 3006 \mathrm{kA}$, and $10000.6 \mathrm{kA}$ leads.

\section{Timescale}

The timescale for the supply of the series leads must fit in with the installation schedule of the LHC machine.

The LHC machine will be installed in the existing LEP tunnel. Installation will start when LEP stops running in October 2000 and will proceed on several fronts in parallel, but will follow a well defined sequence: first general services, then the distribution cryoline and lastly magnet installation. The target start-up date is 2005 , with the intermediate milestone of an octant test in 2003.

The leads will be required for installation in the DFBs. The first DFB will be installed in Summer 2002, so we should have the first production leads for testing before the end of 2001, which implies placing the orders in early 2001 . The specifications should be issued by mid 2000 .

In the meantime, it is planned to install HTS leads in the feedbox of the second "string", a test cell which is to be commissioned in 2000. Successful prototype leads will be used for this purpose, enabling us to get further experience in their installation and operation.

\section{CONCLUSION}

The LHC machine represents a formidable technical challenge both for its designers and the vendors of equipment. The application of HTS technology to the current leads required for this machine provides a unique opportunity for this industry to demonstrate the value of incorporating these new materials into large scale systems. Recent tests confirm that the development of HTS materials has reached a point where, with appropriate engineering effort, it should be possible to make reliable leads and thus meet this particular challenge.

\section{REFERENCES}

[1] The LHC Study Group, "The Large Hadron Collider conceptual design", CERN/AC/95-05, October 1995.

[2] A. Ballarino, "Discendenti di corrente con materiale superconduttore ad alta temperatura per l'alimentazione dei magneti del Large Hadron Collider", PhD thesis, Politecnico di Torino, Turin, 1997.

[3] M. N. Wilson, Superconducting Magnets, Oxford University Press, 1983, pp. 256-272. 